\title{
Roquin 1 inhibits the proliferation of breast cancer cells by inducing G1/S cell cycle arrest via selectively destabilizing the mRNAs of cell cycle-promoting genes
}

Wenbao Lu ${ }^{1 *+}$, Meicen Zhou ${ }^{2+}$, Bing Wang ${ }^{1}$, Xueting Liu ${ }^{1}$ and Bingwei $\mathrm{Li}^{1}$

\begin{abstract}
Background: Dysregulation of cell cycle progression is a common feature of human cancer cells; however, its mechanism remains unclear. This study aims to clarify the role and the underlying mechanisms of Roquin 1 in cell cycle arrest in breast cancer.

Methods: Public cancer databases were analyzed to identify the expression pattern of Roquin 1 in human breast cancers and its association with patient survival. Quantitative real-time PCR and Western blots were performed to detect the expression of Roquin 1 in breast cancer samples and cell lines. Cell counting, MTT assays, flow cytometry, and in vivo analyses were conducted to investigate the effects of Roquin1 on cell proliferation, cell cycle progression and tumor progression. RNA sequencing was applied to identify the differentially expressed genes regulated by Roquin1. RNA immunoprecipitation assay, luciferase reporter assay, mRNA half-life detection, RNA affinity binding assay, and RIP-ChIP were used to explore the molecular mechanisms of Roquin1.

Results: We showed that Roquin1 expression in breast cancer tissues and cell lines was inhibited, and the reduction in Roquin 1 expression was associated with poor overall survival and relapse-free survival of patients with breast cancer. Roquin 1 overexpression inhibited cell proliferation and induced G1/S cell cycle arrest without causing significant apoptosis. In contrast, knockdown of Roquin1 promoted cell growth and cycle progression. Moreover, in vivo induction of Roquin 1 by adenovirus significantly suppressed breast tumor growth and metastasis. Mechanistically, Roquin1 selectively destabilizes cell cycle-promoting genes, including Cyclin D1, Cyclin E1, cyclin dependent kinase 6 (CDK6) and minichromosome maintenance 2 (MCM2), by targeting the stem-loop structure in the $3^{\prime}$ untranslated region (3'UTR) of mRNAs via its ROQ domain, leading to the downregulation of cell cyclepromoting mRNAs.

Conclusions: Our findings demonstrated that Roquin 1 is a novel breast tumor suppressor and could induce G1/S cell cycle arrest by selectively downregulating the expression of cell cycle-promoting genes, which might be a potential molecular target for breast cancer treatment.
\end{abstract}

Keywords: Roquin1, Breast cancer, Cell cycle, Cyclin E1, MCM2, mRNA decay

\footnotetext{
* Correspondence: luwenbao_217@imc.pumc.edu.cn

'Wenbao Lu and Meicen Zhou contributed equally to this work.

'Institute of Microcirculation, Chinese Academy of Medical Sciences \& Peking

Union Medical College, \#69 Dongdan Beidajie, DongCheng District, Beijing

100005, China

Full list of author information is available at the end of the article
}

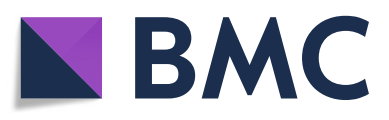

(- The Author(s). 2020 Open Access This article is licensed under a Creative Commons Attribution 4.0 International License, which permits use, sharing, adaptation, distribution and reproduction in any medium or format, as long as you give appropriate credit to the original author(s) and the source, provide a link to the Creative Commons licence, and indicate if changes were made. The images or other third party material in this article are included in the article's Creative Commons licence, unless indicated otherwise in a credit line to the material. If material is not included in the article's Creative Commons licence and your intended use is not permitted by statutory regulation or exceeds the permitted use, you will need to obtain permission directly from the copyright holder. To view a copy of this licence, visit http://creativecommons.org/licenses/by/4.0/ The Creative Commons Public Domain Dedication waiver (http://creativecommons.org/publicdomain/zero/1.0/) applies to the data made available in this article, unless otherwise stated in a credit line to the data. 


\section{Background}

Breast cancer is the most common cancer among women worldwide, and the incidence rates of breast cancer have increased rapidly in China in recent years [1, 2]. Although the death rates of patients have decreased due to the early detection and advanced treatment in recent years, the complex mechanism of tumorigenesis and progression still impede the treatment of breast cancer. Therefore, further elucidation the molecular mechanisms underlying tumorigenesis and progression of breast cancer is still necessary.

Cell cycle dysregulation is a common feature of human cancers, including breast cancer, and is characterized by uncontrolled cell proliferation and cell cycle progression of cancer cells [3-5], which is one reason why tumor cells are capable of unlimited proliferation and resistance to conventional treatments. Although increasing studies have expanded the knowledge on cell cycle regulation, the post-transcriptional mechanism of cell cycle dysregulation in cancer cells, especially through RNA-binding proteins (RBPs), remains unclear.

Roquin1, a Cys-Cys-Cys-His-type RBP encoded by Rc3h1, was initially found to play an important role in immune regulation through its ubiquitin ligase activity [6, 7]. Roquin1 is rapidly induced in T cells upon stimulation by the inflammatory inhibitory factor interleukin-10 [8]. Furthermore, Roquin 1 can suppress autoimmunity by destabilizing the inducible $\mathrm{T}$ cell costimulator (ICOS) mRNA through its ROQ domain [9]. Therefore, Roquin1 is regarded as a regulator of the immune system and orchestrates the differentiation of various immune cells, including follicular helper $\mathrm{T}$ cells (TfHs), Natural killer T (NkT), and regulatory T (Treg) cells [10-12]. Roquin1 deficiency induced the death of mouse embryos and severe autoimmune reactions and enteritis $[13,14]$. Evidence has shown that Roquin1 can induce mRNA decay by binding the stem-loop structure in the 3'UTR of target genes [15-17]. In addition, Roquin1 serves as a regulator of multiple signaling pathways, such as AMP-activated protein kinase (AMPK) [18], NF-кB [19], and PI3K-mTOR [20], to regulate immune responses. Roquin1 is additionally able to regulate microRNA homeostasis [21]. In cancerous TfH cells, the Roquin1 expression level was similar to that in normal $\mathrm{TfH}$ cells [22]. However, it remains unknown whether Roquin1 plays a role in cancer progression.

In this study, we showed that Roquin1 is a potent breast tumor suppressor that induces tumor cell cycle arrest by selectively suppressing the expression of cell cycle-promoting genes, including CCND1, CCNE1, CDK6, and MCM2. Roquin1 expression was reduced in breast cancer tissues and cells, which might contribute to their lack of cell cycle regulation. Ectopic Roquin1 expression induces G1/S cell cycle arrest in breast tumor cells. In contrast, further suppression of Roquin1 expression by shRNAs facilitated tumor cell proliferation and cell cycle progression. Consistent with these in vitro observations, Roquin1 expression in vivo significantly inhibited tumor growth and metastasis. By analyzing a database of human breast tumors [23], we found that low Roquin1 levels in tumor samples were strongly associated with poor survival of luminal A, luminal B, and basal breast cancer patients. Moreover, Roquin1 expression was negatively correlated with CCNE1 and MCM2 in human breast tumors. These results suggested that Roquin1 is a potential tumor suppressor that is involved in regulating cell cycle progression by suppressing cell cycle-promoting genes expression.

\section{Methods}

\section{Animal study}

Six to eight weeks female BALB/c nude mice were bought from the Institute of Laboratory Animal Science, Chinese Academy of Medical Sciences (CAMS) \& Peking Union Medical College (PUMC). The mice were bred in cages with filter tops in a laminar flow hood in pathogen-free condition, with a $12 \mathrm{~h}$ light, $12 \mathrm{~h}$ dark cycle. All experimental procedures were approved by the Experimental Animal Care and Ethics Committee of the Institute of Microcirculation, CAMS \& PUMC. MDA-MB-468/Roquin1-GFP (5 × $10^{6} / 100 \mu \mathrm{L}$ PBS $)$ and MDA-MB-468/GFP cells $\left(5 \times 10^{6} /\right.$ $100 \mu \mathrm{L}$ PBS) were injected subcutaneously into the back of nude mice, respectively. Then, tumor sizes were measured and recorded to draw the tumor growth curve. For tumor treatment with adenovirus, MDA-MB-231cells $\left(3 \times 10^{6}\right.$ / $100 \mu \mathrm{L} \mathrm{PBS})$ were injected into nude mice according to above methods. When tumors reached approximately 5 $\mathrm{mm}$ in diameter, GFP/Roquin1-expressing adenovirus or GFP-expressing control adenovirus (packaged at GeneChem, Shanghai) were injected into the tumors $\left(10^{10} \mathrm{pfu} /\right.$ tumor each time) five times in total. Tumor size was measured by the formula length $\times$ width $\times$ high $\left(\mathrm{mm}^{3}\right)$ in 60 days. Whole lung of nude mice was collected at the end of experiment and immersed in 10\% formaldehyde solution.

\section{Cell lines and plasmids}

The human breast cancer cell line (MDA-MB-231, MDA-MB-468, MCF7 and T47D), human normal mammary epithelial cell lines (MCF-10A and MCF-12A), human lung cancer cell line A549 cell, and human liver cancer cell line (HepG2) cells were obtained from the American Type Culture Collection (ATCC) and cultured in DMEM or RPMI-1640 with 10\% FBS plus 1\% Peni/ Stro, respectively. HEK293 and HEK293T cells were obtained from National Infrastructure of Cell Line Resource (Beijing, China). The human full-length Roquin1 coding sequence (NM_172071) was synthesized, sequenced and inserted into pEGFP-N1 vector at EcoR I and Age I sites. Roquin 1 serial deletion plasmids were generated by inserting the PCR-amplified fragments into pEGFP-N1 
vector at EcoR I and Age I sites. A set of luciferase reporters were constructed by inserting the full-length 3'UTRs of human MCM2, Cyclin D1, and Cyclin E1 or part 3'UTR (1-1440 bp) of CDK6 into the pGL3 control vector (Promega) between Xba I and Fse I sites, respectively. For stem-loop deletion reporters, point mutated and truncated CCNE1-3'UTR (1-256 bp) ( $\triangle$ stem-loop) and MCM2-3'UTR (1-360 bp) ( $\Delta$ stem-loop) were amplified, sequenced, and inserted into pGL3 control vector using Phusion Site-Directed Mutagenesis Kit (Thermo Scientific). For stem-loop insertion constructs, the stemloop sequences of CCNE1 3'UTR (257-276) and MCM2 3'UTR (361-377) were inserted into pGL3- $\beta$-actin ${ }^{3 ' U T R}$ reporter at 555 base pair.

\section{Cell cycle analysis}

$5 \times 10^{5}$ cells were harvested and washed twice with PBS, and then fixed in cold ethanol (70\%). The cells were then stained with propidium iodide $(20 \mu \mathrm{g} / \mathrm{mL})$ and RNase A $(0.2 \mathrm{mg} / \mathrm{mL})$ for $30 \mathrm{~min}$. The stained cells (at least $1 \times 10^{4}$ cells) were analyzed by flow cytometry and the data were analyzed with FlowJo software. All cell cycle analysis was performed in triplicate and repeated at least three times.

\section{RNA-sequencing analysis}

MCF7/Roquin1-GFP, MDA-MB-468/Roquin1-GFP, A549/ Roquin1-GFP, HepG2/Roquin1-GFP, and their control cells (expressing GFP) were cultured for $36 \mathrm{~h}$ and total RNA was extracted using the TRIzol method. RNA sequencing (RNAseq) was completed by Allwegene Technology Inc., Beijing. The cDNA library was then constructed using polymerase chain reaction (PCR) amplification. RNA-seq was performed with the PE150 sequencing strategy using an Illumina second-generation high-throughput sequencing platform. RNA-seq reads with inferior quality or adapters were filtered. Clean read data were processed using Tophat 2 and Cufflinks software to complete the alignment of transcriptomes. Genes not expressed in any sample were excluded from further analysis. Differentially expressed genes and transcripts were then filtered for false discovery rate (FDR)adjusted $P$ values less than or equal to 0.05 .

\section{Gene ontology and Kyoto Encyclopedia of Genes and Genomes (KEGG) pathway analysis}

RNA-seq data were deposited (PRJNA637876). The common up- and -downregulated mRNAs by Roquin1 in tumor cells were classified using the Venn diagram. Gene Ontology (GO) (biological process) and KEGG pathway analyses of commonly downregulated genes were done using DAVI D Bioinformatics Tools and Ingenuity Pathway Analysis.

\section{RNA immunoprecipitation}

Roquin1/GFP fusion protein was expressed in MDAMB-468/Roquin1 cells, and then whole cell lysates were pre-cleared with isotype IgG, followed by incubation with anti-GFP antibody at $4{ }^{\circ} \mathrm{C}$ for $4 \mathrm{~h}$. The protein-RNA complexes were then pulled down by protein $\mathrm{G}$ agarose beads (sc-2002, SantaCruz) and total RNA extracted with TRIzol, followed by detection of cell cycle-promoting genes with RT-PCR.

\section{Luciferase reporter assays}

Luciferase assay was performed as described previously [24]. pGL3 luciferase reporter constructs containing fulllength or segment of 3'UTR of different genes were transfected into HEK293 cells along with Roquin1/GFP, aa 1-441 (contain RING, ROQ, zinc finger domains), aa 441-1133 (contain PRD), aa 174-326 (only contain ROQ domain), and GFP-control constructs, respectively. All transfections were conducted in triplicate and repeated at least three times. The luciferase activity was measured $36 \mathrm{~h}$ after transfection using a Dual-Luciferase Reporter Assay System (Promega).

\section{mRNA stability}

Roquin1/GFP was expressed in MDA-MB-468 cells, and then actinomycin $\mathrm{D}$ (ActD, $5 \mu \mathrm{g} / \mathrm{mL}$ ) and 5, 6dichlorobenzimidazole riboside (DRB, $5 \mu \mathrm{g} / \mathrm{mL}$ ) were added to block de novo RNA synthesis. Total RNA was collected at indicated time points, and the relative mRNA level was analyzed by qPCR. The half-life of mRNA was determined by comparing with the levels of mRNA before adding ActD and DRB. The half-life of different genes in Roquin1 knockdown cells and their corresponding scrambled control cells was also tested as described earlier.

\section{shRNA lentivirus}

Two lentiviral shRNAs (NM_172071.1-3458s1c1; NM_ 172071.1-2032s1c1) targeting human Roquin1 mRNA and two lentiviral shRNAs targeting human MCM2 (NM_0045 26.2-2553s21c1) and Cyclin E1 (NM_001238.1-1149s1c1) were purchased from Sigma. A scramble control shRNA was used as a control. Lentiviral particles were packaged in HEK293T cells by co-transfecting shRNA-pLKO.1, pCMVdR8.2, and pMD2.G constructs. After $48 \mathrm{~h}$, virus supernatants were collected and centrifuged to discard cell debris, and then added to target cells with $1 \mu \mathrm{g} / \mathrm{mL}$ polybrene for overnight. After two rounds infection, the target cells were selected with puromycin $(2.5 \mu \mathrm{g} / \mathrm{mL})$ for 2 weeks, followed by further study.

\section{Hematoxylin and Eosin (H\&E staining)}

Mouse lung tissues were immersed in $10 \%$ formalin for at least 2 weeks, and then stained with $H \& E$ staining. Zeiss Imaging System is used for visualizing of H\&E sections. 


\section{Statistical analysis}

Data in bar graphs represent mean \pm SD of at least three biological repeats. Statistical analysis was performed using Student's t-test by comparing treatment versus vehicle control or otherwise as indicated. $P$-value $<0.05$ was considered to be statistically significant.

\section{Results}

\section{Roquin 1 expression is reduced in breast cancer patients} and is associated with poor survival

Roquin1 expression was first analyzed in a breast tumor database (www.oncomine.org). Low levels of Roquin1 were found in various breast cancers, including breast carcinoma, invasive ductal breast carcinoma, and invasive mixed breast carcinoma, although a moderately high level was found in invasive lobular breast cancer tissues (Fig. 1a; Additional file 1: Figure S1A). Experimentally,
Roquin 1 mRNA expression was significantly reduced in breast tumors compared with normal tissues (Fig. 1b). Roquin1 protein expression was also lower in four randomly selected pairs of breast cancer tissues than in their surrounding normal tissues (Fig. 1c). Moreover, Roquin1 expression was significantly repressed at both the protein (Fig. 1d) and mRNA (Fig. 1e) levels in several human breast cancer cell lines compared with normal mammary gland epithelial cells. Notably, by surveying Roquin1 expression across a gene array dataset [23], we found that low Roquin1 expression in human breast tumor samples was strongly associated with poor overall survival and relapse-free survival of patients (Fig. If and g). Furthermore, the levels of Roquin1 in breast tumors were associated with patient survival in the luminal A, luminal B, and basal-like subsets (Fig. 1h-j). Although no significant correlation was found between Roquin1

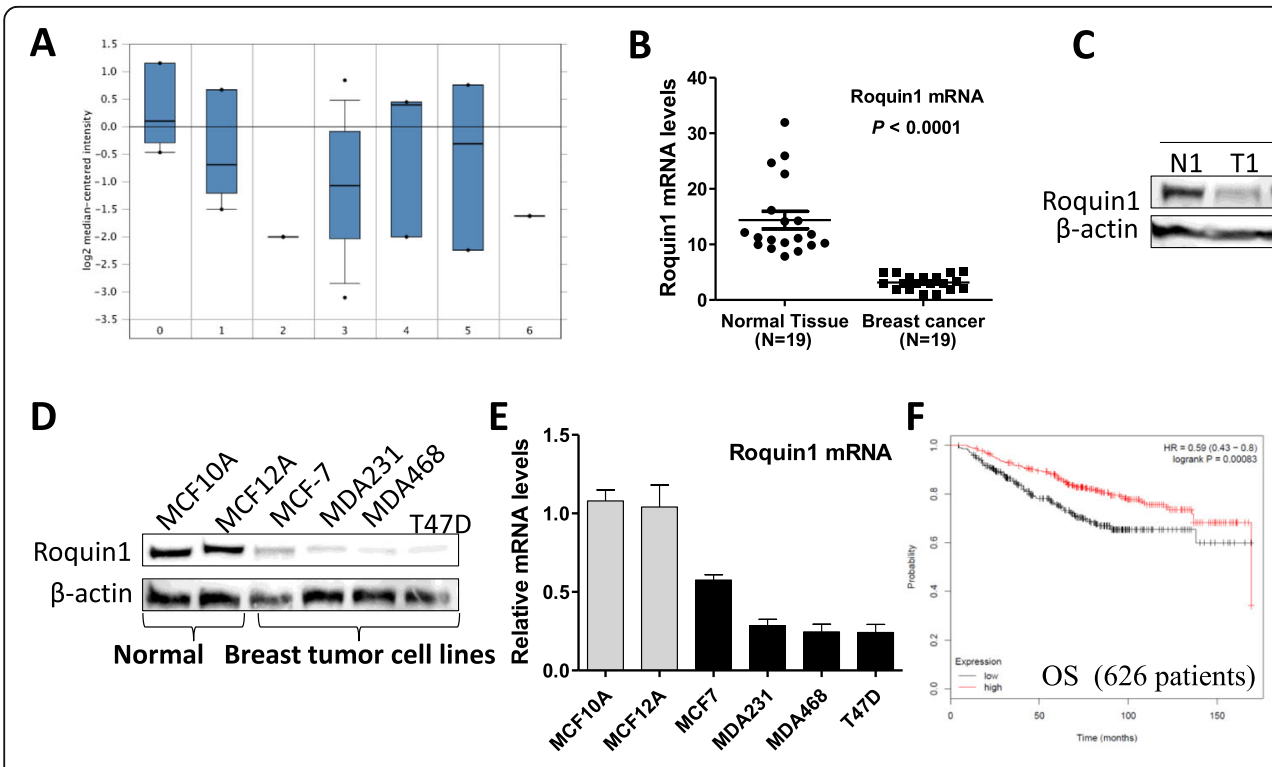

H

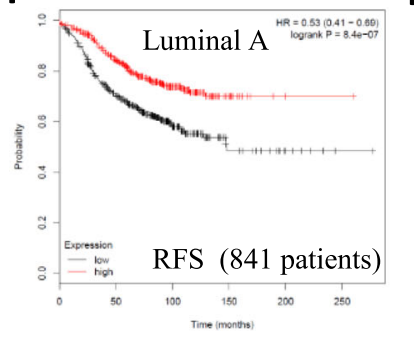

1

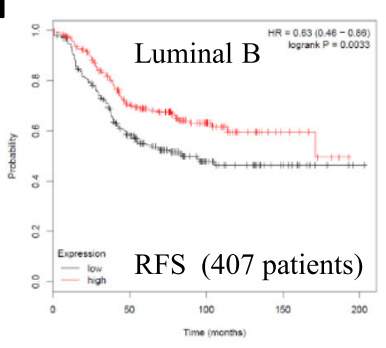

J

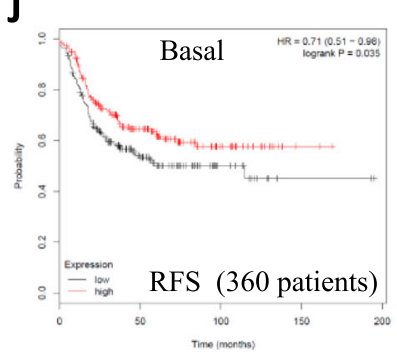

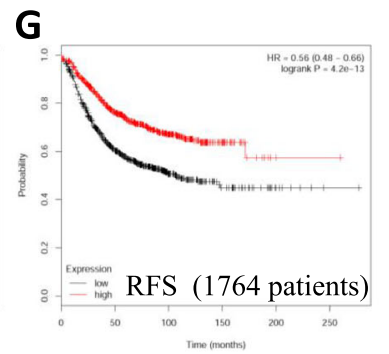

Patients \#

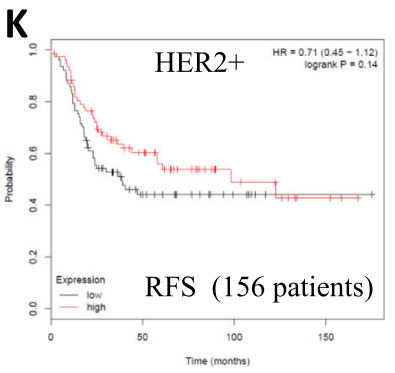

Fig. 1 Roquin 1 expression was reduced in breast cancer patients and was associated with poor survival. a Roquin 1 mRNA expression among normal (0) ( $n=4)$, breast carcinoma (1) $(n=4)$, ductal breast carcinoma in situ (2) $(n=1)$, invasive ductal breast carcinoma (3) $(n=18)$, invasive lobular breast carcinoma (4) $(n=3)$, invasive mixed breast carcinoma (5) $(n=3)$, and male breast carcinoma (6) $(n=1)$. b Roquin 1 expression was measured by qPCR in human breast tumor specimens $(n=19)$ compared with surrounding "normal" breast tissue $(n=19)$. c Roquin1 protein level was measured in human breast tumor tissues and normal mammary gland tissues. $\mathbf{d}$-e Roquin 1 protein (d) and mRNA (e) levels were measured by Western blot analysis and GPCR in human breast tumor cell lines and human normal mammary gland epithelial cell lines, respectively. $\mathbf{f}-\mathbf{g}$ Kaplan-Meier overall survival (f) and relapse-free survival $(\mathbf{g})$ curves of patients with breast tumors having low and high tumor Roquin1 transcripts. h-k Kaplan-Meier relapse-free survival curves of patients with luminal A (h), luminal B (i), basal (j), and Her2+ (k) breast tumor having low and high tumor Roquin1 transcripts 
expression and patient survival in the HER2+ subsets, a similar trend in three other subsets was found (Fig. 1k). These results suggested that Roquin1 was important for the prognosis of patients with breast cancer. In addition, we found that Roquin1 was suppressed in other types of human cancers, including lung cancer, ovarian cancer, gastric cancer, and bladder carcinoma (Additional file 1: Figure S1B-1E). Roquin1 expression levels were also significantly correlated with the prognosis of patients with these cancers and liver cancer (Additional file 1: Figure S1F-1 J), indicating Roquin1 might be clinically predictive for multiple cancers.

Roquin 1 inhibits cell growth by inducing $\mathrm{G} 1 / \mathrm{S}$ phase cell cycle arrest in tumor cells

For analysis of Roquin1 function in breast cancer progression, the Roquin1/GFP fusion protein was expressed in MCF7 and MDA-MB-468 cells and identified by Western blotting (Fig. 2a). When Roquin1 was overexpressed, we found that the proliferation (Fig. 2b, c) and activity (Fig. 2d, e) of the tumor cells were substantially reduced. Similar results were also found in A549 and HepG2 with Roquin1 overexpression (Additional file 1: Figure S2A-2D). To determine whether Roquin1 inhibited cell proliferation by affecting tumor cell cycle progression, we evaluated the effect of Roquin 1 overexpression on the cell cycle by flow cytometry (FCM). The G1 phase percentage of breast tumor cells was significantly increased in the Roquin1overexpressing cancer cells compared with the controls. Moreover, a significant decrease in S phase percentage was detected after Roquin1 overexpression (Fig. 2f, g; Additional file 1: Figure S2E-2F). Similar results were also found in the A549 and HepG2 cells overexpressing Roquin1 (Additional file 1: Figure S2G-2 J). However, the percentage of cells in G2 phase cells did not change consistently among the tumor cells, which might be due to different cell types. These findings suggested that Roquin1 could induce G1/S cell cycle arrest in breast tumor cells.

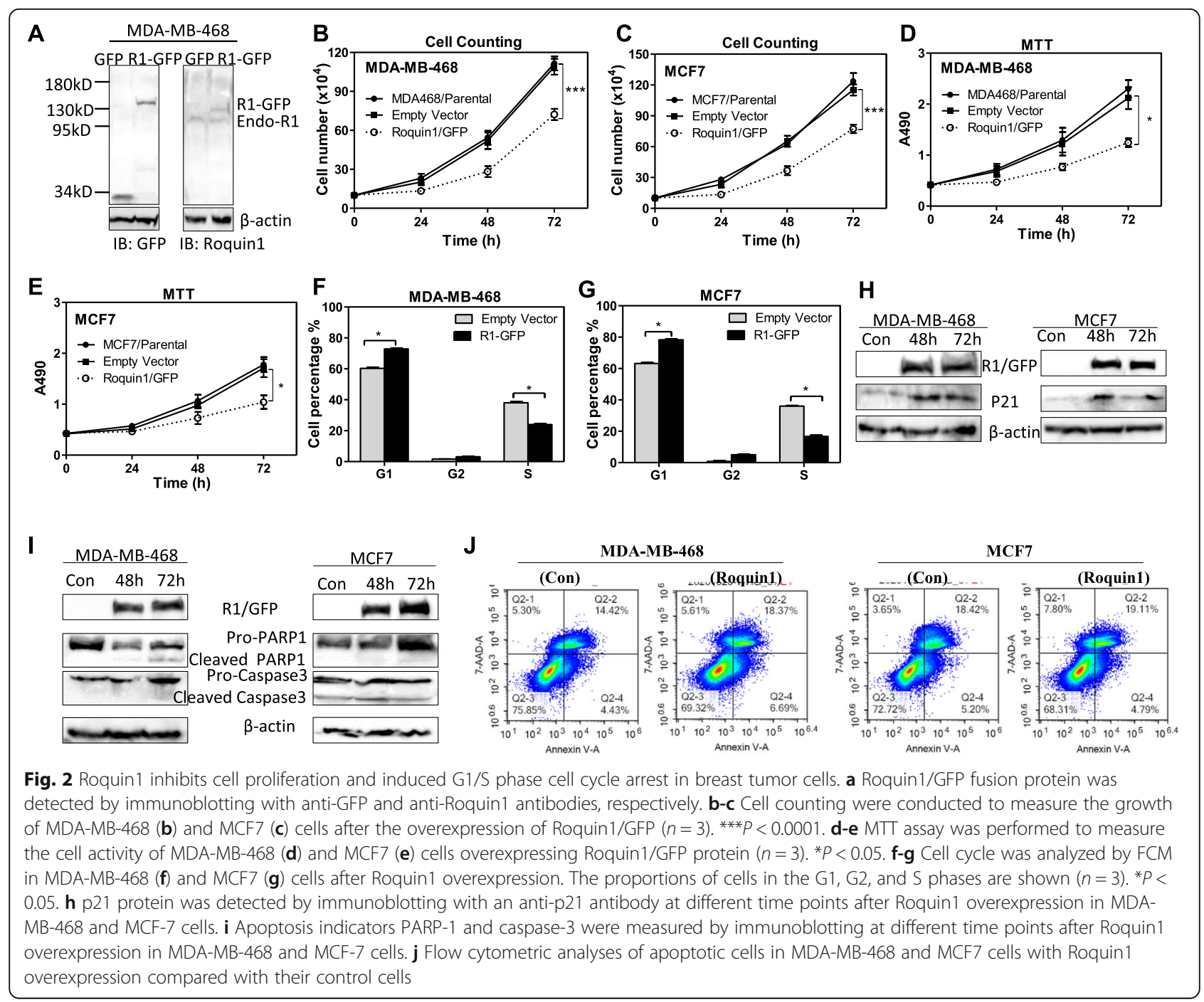


Indeed, the protein levels of p21, a typical cell cycle inhibitor, were induced by Roquin 1 in tumor cells (Fig. $2 \mathrm{~h}$; Additional file 1: Figure S2K-2 L). To determine whether Roquin1 induced apoptosis in breast tumor cells, we detected cleaved caspase 3 and PARP1, two key apoptotic indicators, by Western blotting. Roquin1 could not induce significant cleavage of pro-caspase 3 and pro-PARP1 in breast tumor cells, although cleaved PARP1 was detected in MDA-MB-468 cells $72 \mathrm{~h}$ after Roquin1 overexpression (Fig. 2i). Our FACS data also showed that Roquin1 did not cause cell apoptosis in breast tumor cells (Fig. 2j). Collectively, these data clearly demonstrated that Roquin1 induces G1/S cell cycle arrest in breast tumor cells.

Roquin 1 selectively inhibits the mRNA expression of cell cycle-promoting genes by targeting 3'UTRs

Next, we identified the genes affected by Roquin 1 using RNA-seq in Roquin1-overexpressing MCF7 and MDAMB-468 cells. Venn diagrams showed that 6556 genes were commonly downregulated and 7067 genes were commonly upregulated in two breast tumor cell lines (Additional file 1: Figure S3A). We further focused on the expression of cell cycle-related genes. Interestingly, the genes that promote cell cycle progression, including G1/S transition, G2/M transition, S phase transition, and $M$ phase transition, were suppressed, whereas the genes inhibiting the cell cycle ( $p 21$ and $R b 1)$ were enhanced by Roquin1 in MCF7 (Fig. 3a) and MDA-MB-468 cells (Additional file 1: Figure S3B). Similar trends were also found in A549 and HepG2 cells (Additional file 1: Figure S3C-3D), indicating that Roquin1 could regulate the expression of cell cycle-related genes in tumor cells. Detailed RNA-seq data are summarized in Additional file 3: Table S1. Moreover, the 'cell cycle' pathway was the first of the top 10 signaling pathways significantly enriched in the KEGG pathway analysis of downregulated genes (Fig. 3b). The cell cycle-related terms 'cell division' and 'mitotic nuclear division' were enriched in the Gene Ontology (GO) analysis of downregulated genes (Fig. 3c). These computational analyses further supported our

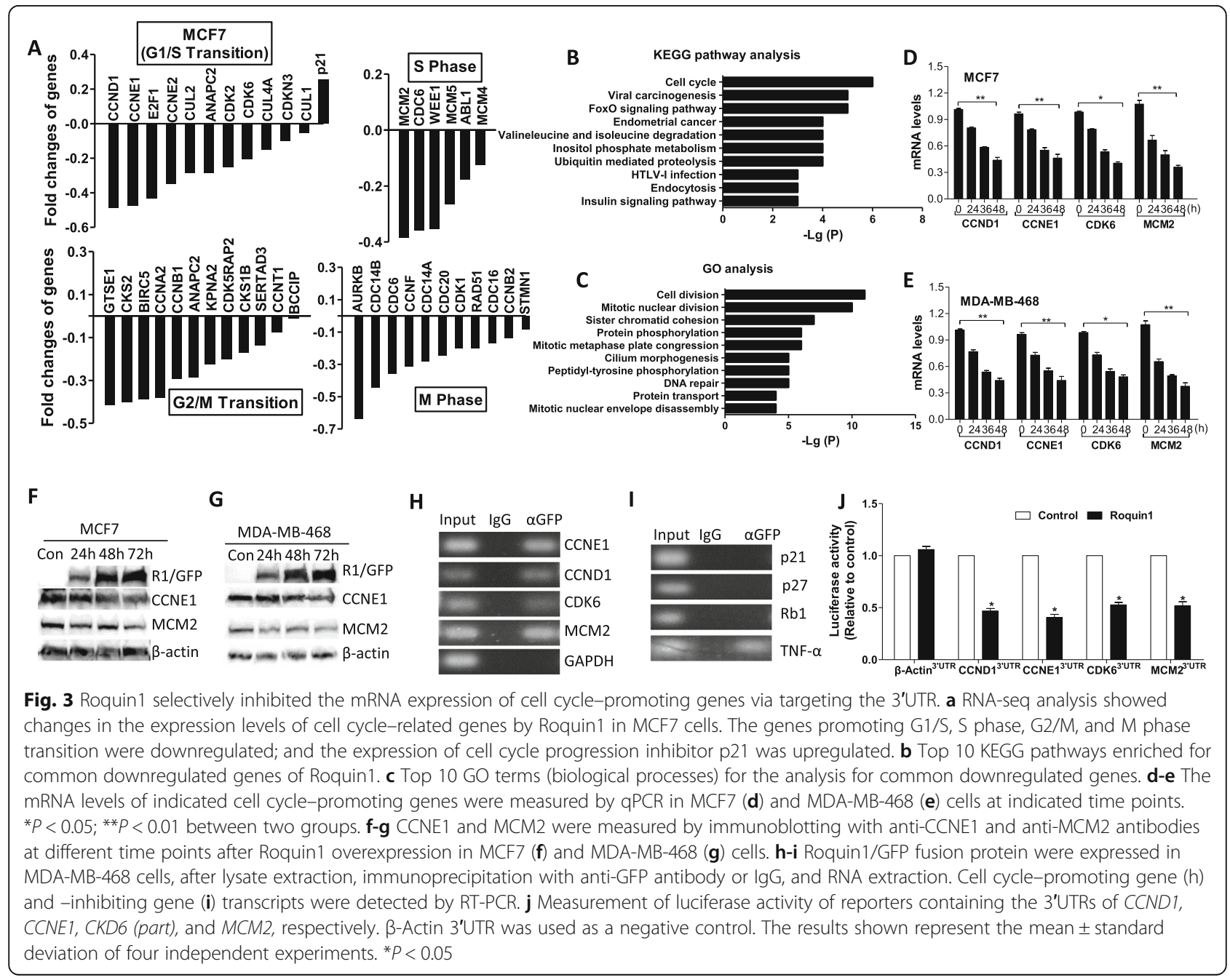


experimental findings. To validate the RNA-seq data, we measured four downregulated cell cycle-promoting genes (CCND1, CCNE1, CDK6, and MCM2) and three upregulated cell cycle-inhibiting genes $(p 21, p 27$, and $R b 1)$ by real-time PCR. The mRNA expression of the four cell cycle-promoting genes was reduced in a time-dependent manner by Roquin1 in tumor cells (Fig. 3d, e). Additionally, the protein levels of CCNE1 and MCM2 were downregulated by Roquin 1 over time (Fig. 3f, g). However, the upregulated cell cycle-inhibiting genes did not exhibit time-dependent changes (Additional file 1: Figure S3E). Notably, no time-dependent changes in the protein levels of p21 were observed in breast tumor cells (Fig. 2h, i). These results confirmed our RNA-seq data. Consistent with the overexpression results, the cell cycle-promoting genes were upregulated in the Roquin $1^{\text {San/San }}$ MEF cells (Additional file 1: Figure S3F) [25], which further strengthened our findings. Taken together, these results indicate that Roquin1 regulates the cell cycle pathway by inhibiting the mRNA expression of cell cycle-promoting genes.

We next examined whether Roquin1 binds to the mRNAs of these cell cycle-promoting genes as an RBP. An RNA pull-down assay was performed with an antiGFP antibody in the Roquin1/GFP-expressing MDA-MB468 cells, followed by detection of the bound mRNAs by RT-PCR. The four cell cycle-promoting genes were amplified by PCR, whereas GAPDH and the cell cycle-inhibiting mRNAs were not amplified (Fig. 3h, i). TNF $\alpha$ was used as a positive control. These results indicated that Roquin1 selectively bound to the cell cycle-promoting genes but not the cell cycle-inhibiting genes. To determine whether mRNA binding was mediated through the 3'UTR, we cloned the 3'UTRs of CCNE1, CCND1, CDK6 (part), and MCM2 downstream of the luciferase gene as previously described [24] and then cotransfected these reporters with the Roquin 1 expression vector and its empty vector into HEK293 cells, followed by the measurement of luciferase activity. As shown in Fig. 3j, Roquin1 significantly inhibited the luciferase activities of all four 3'UTR reporters compared with those of the cells transfected with control vector. The $\beta$-actin 3'UTR was used as a negative control. Collectively, these results suggested that Roquin1 specifically suppressed the mRNA expression of cell cycle-promoting genes by targeting their 3'UTRs.

\section{Roquin 1 destabilizes the mRNAs of cell cycle-promoting genes via the ROQ domain}

We speculated that Roquin1 might reduce cell cyclepromoting genes by destabilizing their mRNAs. For confirmation of this hypothesis, Roquin1/GFP was expressed in MDA-MB-468 cells and then de novo mRNA synthesis was blocked using ActD $(5 \mu \mathrm{g} / \mathrm{mL})$ and DRB $(5 \mu \mathrm{g} /$ $\mathrm{mL}$ ), followed by the measurement of the remaining mRNAs at different time points. The half-lives of indicated cell cycle-promoting mRNAs were shortened approximately 2-fold in Roquin1-overexpressing cells compared with the cells expressing the empty vector (Fig. 4a-d), while the half-lives of cell cycle-inhibiting mRNAs (including $p 21, R b 1$, and $p 27$ ) were barely affected by Roquin1 (Additional file 1: Figure S4A-4C), demonstrating that Roquin1 indeed inhibits cell cyclepromoting genes through mRNA stability.

The Roquin1 protein contains a RING finger, a ROQ domain, a zinc finger (ZF), and a proline-rich domain (PRD), and the ROQ domain is involved in the destabilization of mRNAs [10]. To determine whether the ROQ domain is also responsible for cell cycle-promoting mRNA decay, we generated a series of truncated Roquin 1 mutants, including aa (amino acid) 1-441 containing the RING, ROQ, and ZF domains, aa 441-1133 containing the PRD domain, and aa 174-326 containing the ROQ domain (Fig. 4e), and identified by Western blot analysis (Fig. 4f). Then, the mutants were cotransfected with wild-type (WT) Roquin1 as well as different 3'UTR reporters (Additional file 1: Figure S4D) into HEK293 cells. As shown in Fig. 4g and h, the WT and the mutants aa 1-441 and aa174-326, but not the mutant aa 441-1133, suppressed the mRNA expression of four cell cycle-promoting genes and the luciferase activities of their 3'UTR reporters, which was also consistent with a previous report [26]. In addition, aa 174-326 significantly inhibited the proliferation (Fig. 4i) and cell cycle progression (Fig. 4j; Additional file 1: Figure S4E) of MDA-MB-468 cells, indicating that the ROQ domain in Roquin1 is essential for the induction of breast tumor cell cycle arrest.

\section{Roquin1 knockdown stabilizes cell cycle-promoting gene} transcripts and promotes tumor cell cycle progression

To further confirm the inductive effects of Roquin1 on tumor cell cycle arrest, we suppressed Roquin1 expression with two shRNAs in MDA-MB-231 cells, another triple-negative breast cancer cell line. Roquin1 was reduced by approximately 65 and $74 \%$ by \#1shRNA and \#2shRNA, respectively (Fig. 5a). Although Roquin1 is expressed at low levels in breast tumors, the knockdown of Roquin1 strongly promoted the proliferation and activities of breast tumor cells (Fig. 5b, c) and increased the mRNA expression of cell cycle-promoting genes (Fig. 5d). However, depletion of Roquin1 had no effect on the mRNA levels of p21, Rb1, and p27 (Additional file 1: Figure S5A), again suggesting that Roquin1 directly suppressed the mRNA expression of cell cycle-promoting genes. Next, we examined the effect of Roquin1 knockdown on the half-life of cell cycle-promoting genes. As expected, reduced Roquin 1 significantly prolonged the half-lives of the indicated cell cycle-promoting mRNAs (Fig. 5e-h). Furthermore, we found a reduced percentage of G1 phase cells and an increased percentage of S phase MDA-MB-231 cells after Roquin1 
knockdown (Fig. 5i; Additional file 1: Figure S5B). To confirm whether the cell cycle-promoting genes were involved in the Roquin1-induced cell cycle arrest, we knocked down CCNE1 and MCM2 by shRNA lentivirus in the Roquin1 knockdown MDA-MB-231 cells. Figure 5j shows that these shRNAs effectively knocked down CCNE1 and MCM2 expression. Upon co-knockdown of Roquin1 and CCNE1/MCM2, cell proliferation was closed to that of the scramble control compared to Roquin1 knockdown alone (Fig. 5k). Additionally, the percentage of G1 phase cells was significantly increased compared with that of the group with Roquin1 knockdown alone, and the percentage of $\mathrm{S}$ phase cells significantly decreased (Fig. 5l). Collectively, these results confirmed that Roquin1 repression indeed promotes breast tumor cell cycle progression by stabilizing cell cycle-promoting genes.
Roquin 1 binds to the stem-loop structure of cell cyclepromoting genes for degradation

Roquin 1 is known to degrade target mRNAs by binding to the stem-loop structure [16]. The 3'UTR sequences of four cell cycle-promoting genes were analyzed, and a conserved sequence was identified across species, which could form a similar stem-loop structure (Additional file 1: Figure S6A-6D) using RNAfold WebServer [27]. To investigate the role of the stem-loop structure in Roquin1mediated degradation of cell cycle-promoting mRNAs, we generated deletion constructs by deleting the sequences containing the stem-loop in the 3'UTRs of CCNE1 and MCM2 (Fig. 6a). Then, full-length and deletion reporters with Roquin1 were cotransfected into HEK293 cells, followed by measurement of luciferase activity. Roquin1 significantly inhibited the luciferase activity of the fulllength CCNE1 and MCM2 3'UTRs but not the deletion

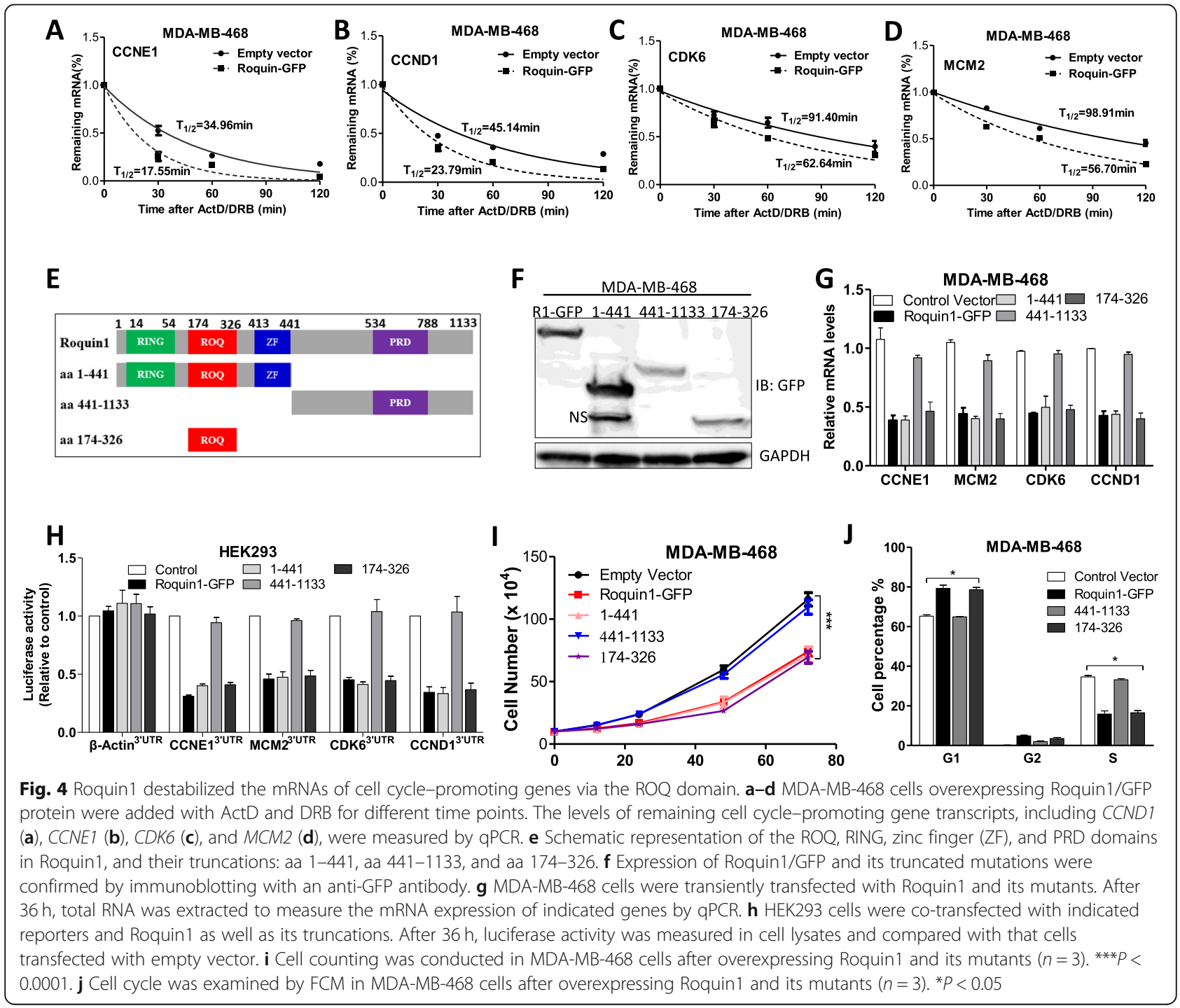




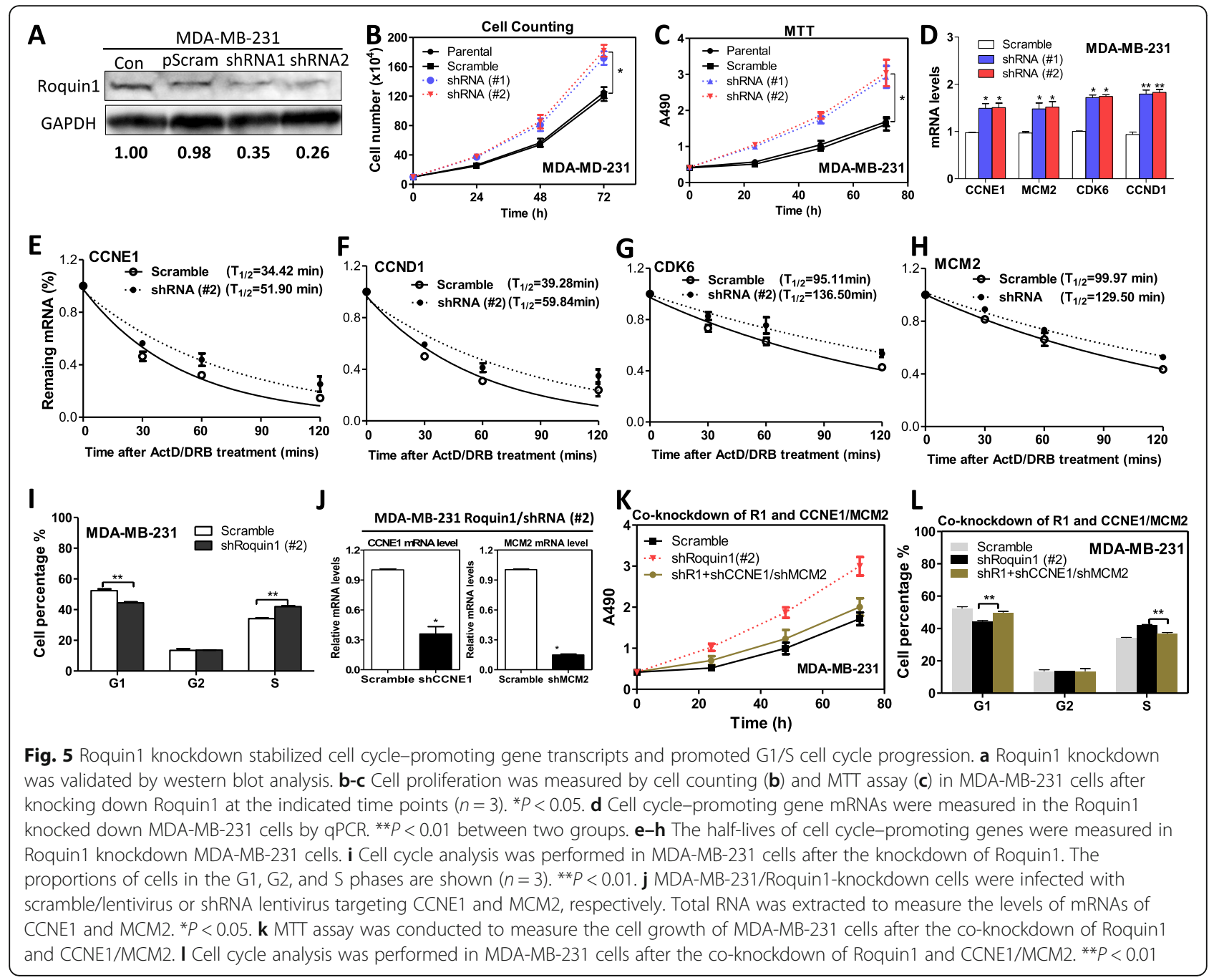

mutant reporters (Fig. 6b). In addition, Roquin1 reduced the activities of the reporters containing human $\beta$-actin 3'UTR with CCNE1 or MCM2 stem-loop structures compared with that in the control group (Fig. 6c, d). These findings indicated that the stem-loop structure was pivotal for Roquin1-mediated cell cycle-promoting mRNAs decay.

To determine the necessity of the stem-loop secondary conformation for mRNA degradation, we generated two 3'UTR mutant reporters of CCNE1 and MCM2; the stem-loop structure of mutant 1 was deleted by replacing two or four nucleotides, and mutant 2 retained the stem-loop structure after replacement of four nucleotides (Fig. 6e). Deletion of the stem-loop structure in the 3'UTRs of CCNE1 and MCM2 (mutant1) allowed them to be completely resistant to Roquin 1 inhibition, while the mutant 2 that maintained the stem-loop structure remained sensitive to Roquin1 suppression (Fig. 6f), indicating that the stem-loop structure in 3'UTRs was critical for cell cycle-promoting mRNAs decay. To further determine whether Roquin 1 physically bound to the stem-loop in the 3'UTRs of CCNE1 and MCM2, we performed an RNA affinity binding assay with biotinlabeled RNA probes. Wild-type RNA probes and mutant probes with the stem-loop structure either disrupted (mutant1) or retained (mutant2) were incubated with lysates of MDA-MB-468 cells expressing the Roquin1/ GFP fusion protein. Then, streptavidin-coated magnetic beads were used for the pulldown assay, followed by Western blot detection with an anti-GFP antibody. The Roquin1/GFP fusion protein was pulled down by wildtype and mutant 2 probes but not by the stem-loop structure-deficient mutant 1 probe (Fig. $6 \mathrm{~g}$ ), indicating that Roquin1 indeed interacted with the stem-loop structure of $C C N E 1$ and $M C M 2$ in vitro. Furthermore, a modified RNA immunoprecipitation-chromatin immunoprecipitation (RIP-ChIP) assay was performed to verify that Roquin1 could bind the stem-loop structure in vivo. The Roquin1/GFP fusion protein was expressed in MDA-MB-468 cells, and the protein-RNA complex 


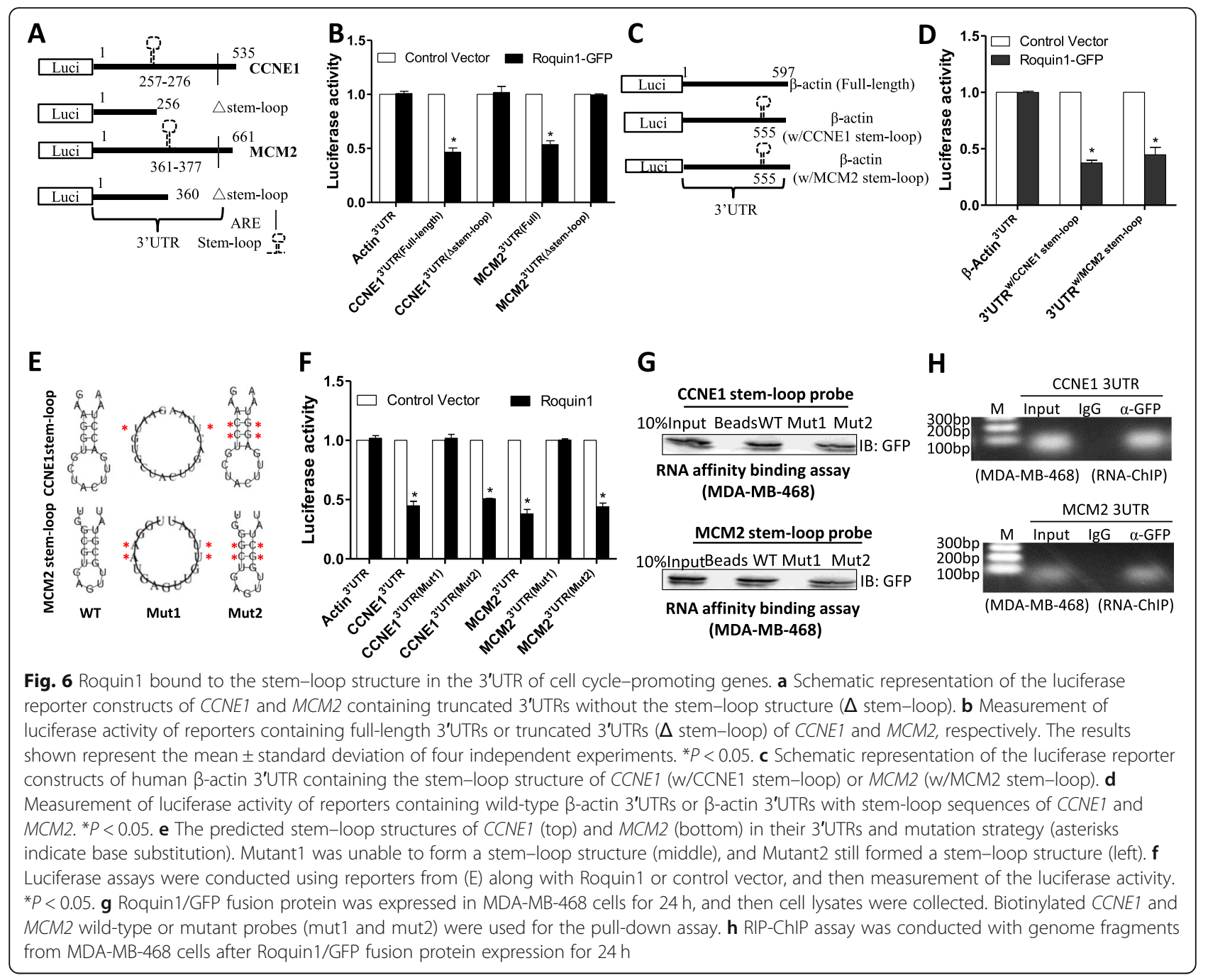

was pulled down by GFP antibody-coated beads after the bound mRNAs were sonicated, followed by amplification of the stem-loop sequences by RT-PCR. As expected, the stem-loop sequences in the 3'UTRs of CCNE1 and $M C M 2$ could be amplified in the GFP antibody pulldown group but not in the group using isotype IgG (Fig. 6h), indicating the binding of Roquin1 to the 3'UTRs of cell cycle-promoting mRNAs in breast tumor cells. Overall, these data demonstrated that Roquin1 recognized and bound to the stem-loop structure in the 3'UTRs of cell cycle-promoting genes for degradation.

Roquin 1 suppresses breast tumor growth and metastasis To determine the inhibitory effect of Roquin 1 on breast cancer progression in vivo, we inoculated MDA-MB-468/ Roquin1-GFP cells (expressing the Roquin1/GFP fusion protein) and MDA-MB-468/GFP cells into the mammary gland fat pads of female nude mice. The growth and sizes of the tumors expressing the Roquin1/GFP fusion protein were significantly reduced compared with those of the control tumors (Fig. 7a, b). Roquin1/GFP fusion protein expression in tumors was confirmed by Western blot analysis (Additional file 1: Figure S7A). Moreover, a significant decrease in the number of metastatic foci (Fig. 7c) and metastatic white nodules (Additional file 1: Figure S7B) was observed in the lung tissues from Roquin1/GFP tumor-bearing mice. To avoid the impacts of the manual manipulation of gene expression and simulate the clinical treatment of breast cancer, we prepared adenoviruses expressing the Roquin1/GFP fusion gene and its control virus (expresses GFP) to treat the established MDA-MB231 breast tumors in nude mice. When tumor mass reached approximately $5 \mathrm{~mm}$ in diameter, $10^{10} \mathrm{pfu}$ of Roquin1/GFP adenovirus in $100 \mu \mathrm{L}$ of PBS and the control adenovirus were injected every other day for five injections in total (Fig. 7d). Two days after injection, the tumors began to shrink and grew slowly, while the tumors treated with control adenovirus continued growing (Fig. 7e). At 


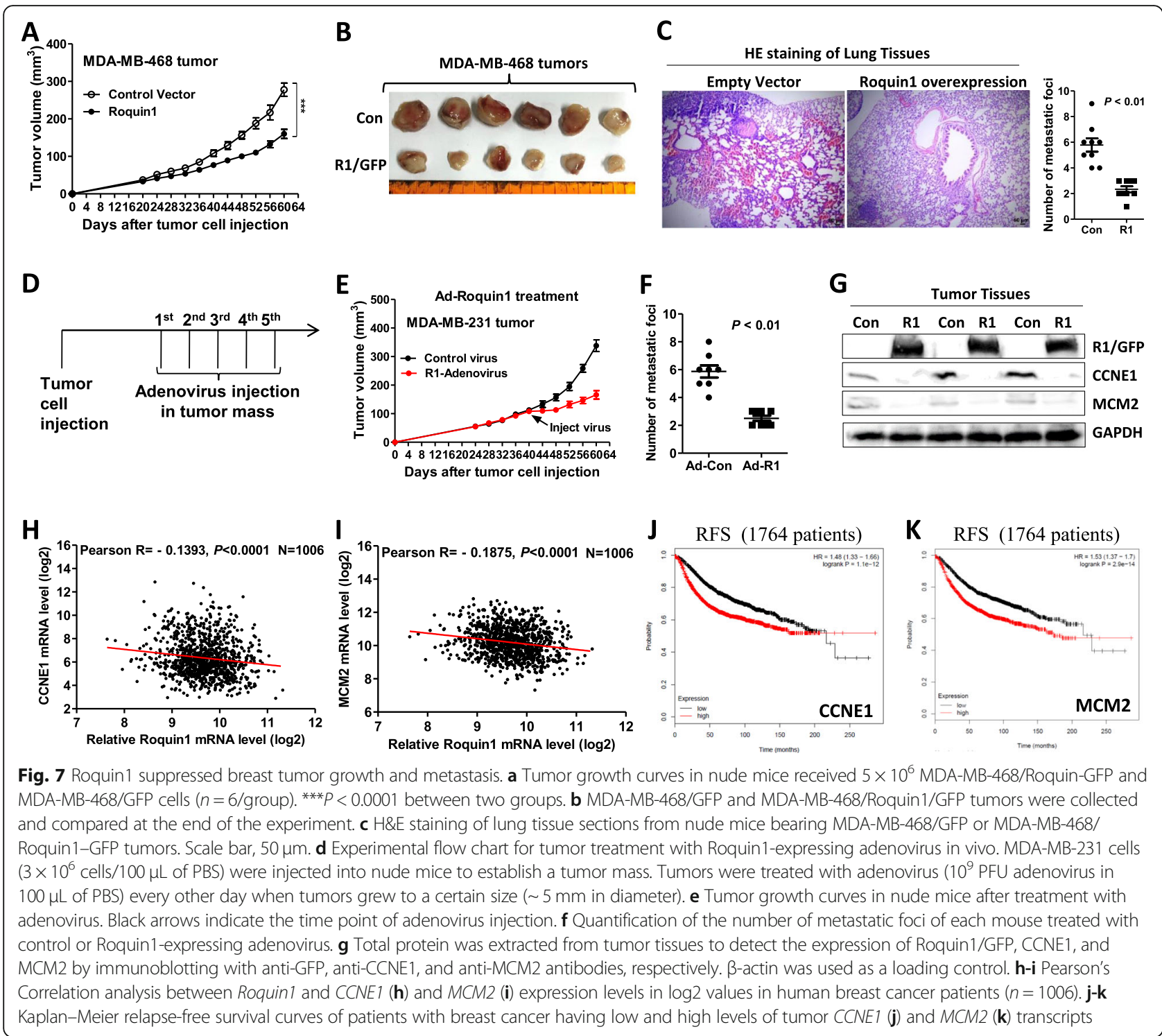

the end of the experiment, the sizes of tumors treated with the Roquin1/GFP adenovirus were significantly smaller than those in the control group (Additional file 1: Figure S7C). Tumor metastasis was also significantly suppressed by Roquin 1 adenovirus treatment (Fig. 7f; Additional file 1: Figure S7D). Consistent with the in vitro results, the protein levels of CCNE1 and MCM2 were also reduced in the Roquin1 adenovirus-treated tumors (Fig. $7 \mathrm{~g}$ ), further confirming that Roquin1 suppressed the expression of cell cycle-promoting genes in vivo. Interestingly, the expression of CCNE1 and MCM2 was also significantly inhibited as Roquin1 increased in 1006 human breast cancer samples (Fig. 7h, i) (Additional file 4: Table S2) (Oncolnc.org/ ). Notably, higher levels of CCNE1 and MCM2 negatively correlated with poor survival of patients with breast cancer (Fig. 7j, k). Conclusively, these findings strongly suggest that Roquin 1 is a promising breast tumor suppressor and that the Roquin1-cell cycle-promoting gene axis might be considered a new therapeutic target for breast tumor treatment in the future.

\section{Discussion}

Currently, the role of Roquin1 in tumor progression has been poorly studied. Analysis of the The Cancer Genome Atlas (TCGA) breast cancer databases showed that Roquin1 was expressed at low levels in human breast cancers. This low-level expression pattern was further confirmed in human breast cancer tissues and cell lines. In addition, low Roquin1 expression in tumors from various subtypes of breast cancer patients was strongly associated with poor survival, indicating the clinical significance of Roquin1. Despite no significant association 
in HER2 + breast cancer, the trend was similar to that of other subtypes. Besides, the levels of Roquin1 in several types of human cancers were associated with patient survival, indicating that Roquin1 might be important for the prognosis of multiple types of human cancers.

We initially focused on the changes of cytokines secreted by tumor cells following Roquin1 overexpression. Unexpectedly, we found that Roquin1 significantly suppressed cell proliferation and induced cell cycle arrest in vitro. To our knowledge, the relationship between Roquin 1 and cell cycle progression has not been reported to date, especially in breast tumor progression. Using FCM, we found that Roquin1 could significantly inhibit the G1/S transition in breast cancer cells as well as lung and liver cancer cells. However, we did not observe obviously cell apoptosis after Roquin1 overexpression, which was different from the apoptotic induction role of another RBP, monocyte chemotactic protein-induced protein 1 (MCPIP1), in breast cancer progression [24]. Cell cycle arrest is triggered when the balance between cell cycle-promoting and cell cycle-inhibiting genes is disrupted. Our results clearly demonstrated that most of the genes that promoted cell cycle progression were downregulated by Roquin1, while those that inhibited cell cycle progression were upregulated. Indeed, four cell cycle-promoting genes, but not the cell cycle-inhibiting genes, were confirmed to be suppressed in a time-dependent manner. Selective targeting of Roquin 1 to cell cycle-promoting mRNAs but not to cycle-inhibiting mRNAs might trigger an imbalance between cell cycle-promoting and inhibiting genes in tumor cells. Roquin 1 also inhibited cell cycle progression in human liver cancer cells and lung cancer cells, indicating that Roquin1 might elicit an antitumor response in a wide range of human cancers.

Among the Roquin1 target genes, CCNE1, CCND1, and $C D K 6$ are involved mainly in the G1/S transition [28-30], while MCM2 is important for DNA replication and the $S$ phase transition of the cell cycle [31]. The broad targets of Roquin1 in the cell cycle pathway suggest that Roquin1 induces cell cycle arrest by targeting multiple molecules in breast tumor cells. We showed that Roquin1 suppresses the expression of these genes at the post-transcriptional level by enhancing mRNA degradation. The 3'UTR is important for the post-transcriptional regulation of genes. Many RBPs, including human antigen $\mathrm{R}(\mathrm{HuR})$ [32], tristetraprolin (TTP) [33], and MCPIP1, have been reported to regulate mRNA stability by the 3'UTR. Indeed, Roquin1 inhibits luciferase activity through the 3'UTRs of cell cycle-promoting genes. It has been reported that the ROQ domain is essential for Roquin1-mediated mRNA degradation and immune regulatory effects [34]. We demonstrated that the ROQ domain is also required for the expression of cell cycle-promoting genes and cell cycle arrest induction by mutating the domains of Roquin 1 .

Many elements responsible for mRNA degradation are primarily localized in the 3'UTR, such as ARE, GU-rich element (GRE), and stem-loop structure [35-37]. Roquin is known to recruit other deadenylases to the 3'UTR for RNA decay by recognizing and binding the stem-loop structure [38]. Our results demonstrated that the stem-loop structure but not the ARE in the 3'UTRs is required for Roquin1-mediated cell cycle-promoting mRNAs decay. Roquin1 has been shown to bind a constitutive decay element (CDE) in the 3'UTR of TNF $\alpha$ mRNA, and this CDE can fold into a stem-loop structure [39]. Indeed, we found that a conserved consensus sequence was shared among different specifies in four cell cycle-promoting genes, and these sequences could fold into a stem-loop structure. Strikingly, no common stem-loop sequences were identified among the four cell cycle-promoting genes, which supports our hypothesis that Roquin1 mainly recognizes the secondary structure instead of the linear sequence in the 3'UTR, which was also consistent with previous findings [15].

Finally, we proposed a model to elucidate the potential role of Roquin1 in the suppression of cell cycle progression (Additional file 1: Figure S7E). Roquin1 selectively targets the mRNAs of cell cycle-promoting gene for degradation via its ROQ domain by binding to the stem-loop structures in the 3'UTRs. As an RBP located in the cytoplasm, Roquin1 can regulate cell cycle progression by balancing the expression of cell cyclerelated genes in tumor cells. Moreover, Roquin1 expression was significantly negatively correlated with the expression of cell cycle-promoting genes in human breast tumors, further demonstrating the biological relevance of Roquin1 and the suppression of breast cancer. Our findings provide a novel regulator for the cell cycle signaling pathway and identify new target genes of Roquin1.

\section{Conclusions}

In summary, this study demonstrates that Roquin 1 implicates in regulating the growth and metastasis of breast cancer by inhibiting cell cycle progression and proliferation. Roquin1 disrupts the balance of the cell cycle signaling pathway by directly binding and destabilizing cell cyclepromoting genes via the ROQ domain, which ultimately induced G1/S cell cycle arrest in cancer cells. Notably, low Roquin1 expression in breast tumors is strongly associated with poor survival of patients with breast cancer. Therefore, Roquin1 might be a new cell cycle suppressor in breast cancer, which could be a promising molecular target for tumor treatment. 


\section{Supplementary Information}

The online version contains supplementary material available at https://doi. org/10.1186/s13046-020-01766-w.

Additional file 1: Figure S1. Roquin 1 expression is reduced in several human cancers, and positively associated with patient survival. a Comparison of Roquin 1 mRNA expression between normal $(0)(n=7)$ and ductal breast carcinoma (1) $(n=40)$. b Comparison of Roquin 1 mRNA expression among normal $(0)(n=65)$, large cell lung carcinoma (1) $(n=19)$, lung adenocarcinoma (2) $(n=45)$, and squamous cell lung carcinoma (3) ( $n=27)$. c Comparison of Roquin1 mRNA expression among normal $(0)(n=5)$, ovarian clear cell large cell adenocarcinoma (1) $(n=7)$, ovarian endometrioid adenocarcinoma (2) $(n=9)$, ovarian mucinous adenocarcinoma (3) $(n=9)$, and ovarian serous adenocarcinoma (4) $(n=20)$. d Comparison of Roquin 1 mRNA expression in normal (0) $(n=31)$, diffuse gastric adenocarcinoma (1) $(n=6)$, and gastric adenocarcinoma (2) $(n=2)$. e Comparison of Roquin 1 mRNA expression in normal $(0)(n=3)$, bladder cancer (1) $(n=$ 3), bladder squamous cell carcinoma (2) $(n=1)$, bladder urothelial carcinoma (3) $(n=3)$, infiltrating bladder urothelial carcinoma (4) $(n=$ 34), bladder papillary urothelial carcinoma (5) $(n=1)$, and superficial bladder cancer (6) ( $n=17)$. f-j Kaplan-Meier overall survival curve of patients with lung cancer ( $)$, ovarian cancer (g), gastric cancer (h), bladder carcinoma (i), and liver cancer (j) having low and high tumor Roquin1 transcripts. Figure S2. Roquin1 inhibits cell proliferation and induces G1/S phase cell cycle arrest in tumor cells. a-b Cell counting was carried out every $24 \mathrm{~h}$ in A549 (a) and HepG2 (b) cells with Roquin1/GFP overexpression. ${ }^{* *} P<0.001$. c-d MTT assay was performed in A549 and HepG2 cells to measure cell proliferation after Roquin1 overexpression. ${ }^{*} P<0.05$. e-h Representative cell cycle histograms showing cell cycle analyses of MDA-MB-468 (e), MCF7 (f), A549 (g), and HepG2 (h) cells after Roquin 1 overexpression. i-j Cell cycle analysis was carried out in A549 (i) and HepG2 (j) cells after Roquin1 overexpression, and the percentages of different cell phases were quantified. ${ }^{* *} P<0.01$. k-I The protein level of cell cycle inhibitor p21 was measured by immunoblotting with an anti-p21 antibody at different time points after Roquin1 overexpression in A549 ( $k$ ) and HepG2 (I) cells. $\beta$-actin was used as a loading control. Figure S3. Roquin 1 selectively inhibits the mRNA expression of cell cycle-promoting genes. a Venn diagrams showing the common down-regulated (upper) and up-regulated genes (bottom) by Roquin 1 in breast tumor cells. b-d The expression levels of cell cycle-related genes affected by Roquin1 were analyzed by RNA-seq in MDA-MB-468 (b), A549 (c), and HepG2 (d) cells. The expression of genes promoting G1/S, S phase, G2/M, and M phase transition was downregulated; and the expression of cell cycle-inhibiting genes were upregulated. e The mRNA expression levels of indicated cell cycle-inhibiting genes were measured by qPCR in MDA-MB-468 and MCF7 cells at different time points, including $p 21, p 27, p 16$, and $R b 1 . f$ Cell cycle-related genes were regulated by Roquin 1 in Roquin $1^{\text {san/san }} \mathrm{MEF}$ cells. The expression of genes promoting G1/S, S phase, G2/M, and M phase transition were upregulated. Figure S4. Roquin1 destabilizes the mRNAs of cell cyclepromoting genes via the ROQ domain. a-c The half-lives of cell cycleinhibiting genes, including p21 (a), p16 (b), and p27 (c) were measured by $q P C R$ in Roquin1-expressing MDA-MB-468 cells. d Schematic representation of the luciferase reporter constructs containing $3^{\prime} U T R s$ sequences of CCNE1, CCND1, CDK6 (part), and MCM2. e Representative cell cycle histograms showing cell cycle analyses of MDA-MB-468 cells after overexpression of Roquin1/GFP, aa 441-1131, and aa 174-326 truncated mutations. Figure S5. Knocking down Roquin1 enhances breast tumor cell cycle progression. a The mRNA expression levels of indicated cell cycle-inhibiting genes were measured after Roquin 1 knockdown by infecting lentivirus expressing shRNA/Scramble and shRNA/Roquin1 by $\mathrm{APCR}$ in MDA-MB-231 cells. b Representative cell cycle histograms showing cell cycle analyses of MDA-MB-231 cells after knocking down Roquin 1. Figure S6. Putative stem-loop structure in the $3^{\prime} U T R s$ of cell cycle-promoting genes. a-d The 3'UTR sequences from different species for each cell cycle-promoting gene, including CCNE1 (a), MCM2 (b), CDK6 (c), and CCND1 (d), was aligned using DNAMAN software. The stem-loop sequences were predicted by RNAfold web server to fold a secondary stem-loop structure (right) and indicated by red box. Figure
S7. Roquin 1 suppresses breast tumor growth and metastasis. a Total protein was extracted from tumor tissues and used to detect Roquin1/ GFP expression by immunoblotting with an anti-GFP antibody. b Whole lungs from nude mouse bearing MDA-MB-468/GFP or MDA-MB-468/ Roquin1/GFP tumors was collected and compared. c MDA-MB-231 tumors treated with control adenovirus (Ad-GFP) or Roquin1-expressing adenovirus (Ad-R1/GFP). d H\&E staining of lung sections of tumorbearing mice treated with control adenovirus or Roquin1-expressing adenovirus. Scale bar, 50 mm. e A proposed work model of cell cyclepromoting genes regulation by Roquin 1 .

\section{Additional file 2.}

Additional file 3: Supplemental Table 1. RNA-seq analysis of human tumor cells overexpressing Roquin1.

\section{Additional file 4.}

Additional file 5: Supplementary Table S3. List of primer and RNAEMSA probes sequences used in this study.

\section{Abbreviations}

MTT: Thiazolyl Blue Tetrazolium Bromide; FCM: Flow cytometry; RIP-ChIP: RNA immunoprecipitation-chromatin immunoprecipitation; CCND1: Cyclin D1; CCNE1: Cyclin E1; CDK6: Cyclin dependent kinase 6; MCM2: Minichromosome maintenance 2; 3'UTR: 3' untranslated region; RBP: RNA-binding protein; RING: Really Interesting New Gene; ZF: Zinc finger; ROQ: ROQ domain containing RNA binding activity; ATCC: American Type Culture Collection; RPMI 1640: Roswell Park Memorial Institute 1640; shRNA: Short hairpin RNA; DMEM: Dulbecco's Modified Eagle's Medium; DRB: 5, 6-

dichlorobenzimidazole riboside; ActD: Actinomycin D; ARE: AU-rich element; MEF: Mouse Embryonic Fibroblast

\section{Acknowledgements}

We thank all individuals who take part in this research.

\section{Authors' contributions}

WBL and MCZ were in responsible for the design of the study and wrote the manuscript; WBL participated in the RNA-seq analysis and bioinformatic analysis; MCZ performed qPCR and Western blot experiments; MCZ and BW contributed to culture of cells and in vitro cell assays; WBL, MCZ and BWL contributed to perform the study of the mechanism investigation; WBL, $M C Z$, and BWL were in charge of the animal experiments; XTL helped conduct the animal study. All of the authors reviewed the manuscript before submission and approved the final manuscript.

\section{Funding}

This work was supported by grants from the National Natural Science Foundation of China (NSFC) (Grant Number: 81702769), CAMS Innovation Fund for Medical Science (No. 2017-I2M-1-016), and the PUMC Youth Fund (Grant Number: 3332017105).

\section{Availability of data and materials}

All data generated or analyzed during this study are included either in this article or in the supplementary information files.

\section{Ethics approval and consent to participate}

The study was approved by the Medical Ethics Committee of the Institute of Microcirculation, CAMS \& PUMC

\section{Consent for publication}

All authors agree on publication of the results of the present manuscript.

\section{Competing interests}

The authors declare that they have no competing interests.

\section{Author details}

${ }^{1}$ Institute of Microcirculation, Chinese Academy of Medical Sciences \& Peking Union Medical College, \#69 Dongdan Beidajie, DongCheng District, Beijing 100005, China. ${ }^{2}$ Department of Endocrinology, Beijing Jishuitan Hospatial, The 4th Clinical Medical College of Peking University, Beijing 100035, China. 
Received: 4 August 2020 Accepted: 5 November 2020

Published online: 23 November 2020

\section{References}

1. Bray F, Ferlay J, Soerjomataram I, Siegel RL, Torre LA, Jemal A. Global cancer statistics 2018: GLOBOCAN estimates of incidence and mortality worldwide for 36 cancers in 185 countries. CA Cancer J Clin. 2018;68(6):394-424.

2. Feng RM, Zong YN, Cao SM, Xu RH. Current cancer situation in China: good or bad news from the 2018 global Cancer statistics? Cancer Commun (Lond). 2019;39(1):22.

3. Bai J, Li Y, Zhang G. Cell cycle regulation and anticancer drug discovery. Cancer Biol Med. 2017;14(4):348-62.

4. Thu KL, Soria-Bretones I, Mak TW, Cescon DW. Targeting the cell cycle in breast cancer: towards the next phase. Cell Cycle. 2018;17(15):1871-85.

5. Pandey K, An HJ, Kim SK, Lee SA, Kim S, Lim SM, et al. Molecular mechanisms of resistance to CDK4/6 inhibitors in breast cancer: a review. Int J Cancer. 2019;145(5):1179-88.

6. Vinuesa CG, Cook MC, Angelucci C, Athanasopoulos V, Rui L, Hill KM, et al. A RING-type ubiquitin ligase family member required to repress follicular helper T cells and autoimmunity. Nature. 2005:435(7041):452-8.

7. Yu D, Tan AH, Hu X, Athanasopoulos V, Simpson N, Silva DG, et al. Roquin represses autoimmunity by limiting inducible T-cell co-stimulator messenger RNA. Nature. 2007;450(7167):299-303.

8. Schaefer JS, Montufar-Solis D, Klein JR. A role for IL-10 in the transcriptional regulation of Roquin-1. Gene. 2014;549(1):134-40.

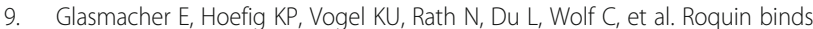
inducible costimulator mRNA and effectors of mRNA decay to induce microRNA-independent post-transcriptional repression. Nat Immunol. 2010; 11(8):725-33.

10. Heissmeyer $\mathrm{V}$, Vogel KU. Molecular control of Tfh-cell differentiation by Roquin family proteins. Immunol Rev. 2013;253(1):273-89.

11. Drees C, Vahl JC, Bortoluzzi S, Heger KD, Fischer JC, Wunderlich FT, et al. Roquin Paralogs differentially regulate functional NKT cell subsets. J Immunol. 2017;198(7):2747-59.

12. Lee SY, Lee SH, Seo HB, Ryu JG, Jung K, Choi JW, et al. Inhibition of IL17 ameliorates systemic lupus erythematosus in Roquin (san/san) mice through regulating the balance of TFH cells, GC B cells. Treg Breg Sci Rep. 2019;9(1):5227.

13. Bertossi A, Aichinger $M$, Sansonetti $P$, Lech $M$, Neff $F$, Pal $M$, et al. Loss of Roquin induces early death and immune deregulation but not autoimmunity. J Exp Med. 2011;208(9):1749-56.

14. Schaefer JS, Montufar-Solis D, Nakra N, Vigneswaran N, Klein JR. Small intestine inflammation in Roquin-mutant and Roquin-deficient mice. PLoS One. 2013;8(2):e56436.

15. Schuetz A, Murakawa Y, Rosenbaum E, Landthaler M, Heinemann U. Roquin binding to target mRNAs involves a winged helix-turn-helix motif. Nat Commun. 2014:5:5701

16. Janowski R, Heinz GA, Schlundt A, Wommelsdorf N, Brenner S, Gruber AR et al. Roquin recognizes a non-canonical hexaloop structure in the $3^{\prime}-U T R$ of Ox40. Nat Commun. 2016:7:11032.

17. Braun J, Fischer S, Xu ZZ, Sun H, Ghoneim DH, Gimbel AT, et al. Identification of new high affinity targets for Roquin based on structural conservation. Nucleic Acids Res. 2018;46(22):12109-25.

18. Ramiscal RR, Parish IA, Lee-Young RS, Babon JJ, Blagih J, Pratama A, et al. Attenuation of AMPK signaling by ROQUIN promotes T follicular helper cell formation. Elife. 2015;4(e08698):1-22.

19. Murakawa $Y$, Hinz M, Mothes J, Schuetz A, Uhl M, Wyler E, et al. RC3H post-transcriptionally regulates $\mathrm{A} 20 \mathrm{mRNA}$ and modulates the activity of the IKK/NF-kappaB pathway. Nat Commun. 2015:6:7367.

20. Essig K, Hu D, Guimaraes JC, Alterauge D, Edelmann S, Raj T, et al. Roquin suppresses the PI3K-mTOR signaling pathway to inhibit T helper cell differentiation and conversion of Treg to Tfr cells. Immunity. 2017;47(6): 1067-82 e12.

21. Srivastava M, Duan G, Kershaw NJ, Athanasopoulos V, Yeo JH, Ose T, et al. Roquin binds microRNA-146a and Argonaute2 to regulate microRNA homeostasis. Nat Commun. 2015:6:6253.

22. Auguste T, Travert M, Tarte K, Ame-Thomas P, Artchounin C, Martin-Garcia $\mathrm{N}$, et al. ROQUIN/RC3H1 alterations are not found in angioimmunoblastic Tcell lymphoma. PLoS One. 2013;8(6):e64536.

23. Gyorffy B, Lanczky A, Eklund AC, Denkert C, Budczies J, Li Q, et al. An online survival analysis tool to rapidly assess the effect of 22,277 genes on breast cancer prognosis using microarray data of 1,809 patients. Breast Cancer Res Treat. 2010;123(3):725-31.

24. Lu W, Ning H, Gu L, Peng H, Wang Q, Hou R, et al. MCPIP1 selectively destabilizes transcripts associated with an Antiapoptotic gene expression program in breast Cancer cells that can elicit complete tumor regression. Cancer Res. 2016;76(6):1429-40.

25. Mino T, Murakawa Y, Fukao A, Vandenbon A, Wessels HH, Ori D, et al. Regnase-1 and Roquin regulate a common element in inflammatory mRNAs by spatiotemporally distinct mechanisms. Cell. 2015;161(5):1058-73.

26. Schlundt A, Heinz GA, Janowski R, Geerlof A, Stehle R, Heissmeyer V, et al. Structural basis for RNA recognition in roquin-mediated post-transcriptional gene regulation. Nat Struct Mol Biol. 2014;21(8):671-8.

27. Hofacker IL. Vienna RNA secondary structure server. Nucleic Acids Res. 2003; 31(13):3429-31.

28. Wu Y, Zheng Q, Li Y, Wang G, Gao S, Zhang X, et al. Metformin targets a YAP1-TEAD4 complex via AMPKalpha to regulate CCNE1/2 in bladder cancer cells. J Exp Clin Cancer Res. 2019;38(1):376.

29. Tyagi N, Deshmukh SK, Srivastava SK, Azim S, Ahmad A, Al-Ghadhban A, et al. ETV4 facilitates cell-cycle progression in pancreatic cells through transcriptional regulation of Cyclin D1. Mol Cancer Res. 2018;16(2):187-96.

30. Ingham M, Schwartz GK. Cell-cycle therapeutics come of age. J Clin Oncol. 2017:35(25):2949-59.

31. Zhang $X$, Teng $Y$, Yang $F$, Wang $M$, Hong $X$, Ye LG, et al. MCM2 is a therapeutic target of lovastatin in human non-small cell lung carcinomas. Oncol Rep. 2015;33(5):2599-605.

32. Ouhara K, Munenaga S, Kajiya M, Takeda K, Matsuda S, Sato Y, et al. The induced RNA-binding protein, HuR, targets 3'-UTR region of IL-6 mRNA and enhances its stabilization in periodontitis. Clin Exp Immunol. 2018;192(3):325-36.

33. Kim DJ, Vo MT, Choi SH, Lee JH, Jeong SY, Hong CH, et al. Tristetraprolinmediated hexokinase 2 expression regulation contributes to glycolysis in cancer cells. Mol Biol Cell. 2019;30(5):542-53.

34. Tan D, Zhou M, Kiledjian M, Tong $L$. The ROQ domain of Roquin recognizes mRNA constitutive-decay element and double-stranded RNA. Nat Struct Mo Biol. 2014;21(8):679-85.

35. Khabar KS. Post-transcriptional control during chronic inflammation and cancer: a focus on AU-rich elements. Cell Mol Life Sci. 2010;67(17):2937-55.

36. Vlasova IA, Tahoe NM, Fan D, Larsson O, Rattenbacher B, Sternjohn JR, et al. Conserved GU-rich elements mediate mRNA decay by binding to CUGbinding protein 1. Mol Cell. 2008;29(2):263-70.

37. Li M, Cao W, Liu H, Zhang W, Liu X, Cai Z, et al. MCPIP1 downregulates $\mathrm{IL}-2$ expression through an ARE-independent pathway. PLoS One. 2012;7(11):e49841.

38. Sgromo A, Raisch T, Bawankar P, Bhandari D, Chen $Y$, Kuzuoglu-Ozturk $D$, et al. A CAF40-binding motif facilitates recruitment of the CCR4-NOT complex to mRNAs targeted by Drosophila Roquin. Nat Commun. 2017; 8:14307.

39. Leppek K, Schott J, Reitter S, Poetz F, Hammond MC, Stoecklin G. Roquin promotes constitutive mRNA decay via a conserved class of stem-loop recognition motifs. Cell. 2013;153(4):869-81.

\section{Publisher's Note}

Springer Nature remains neutral with regard to jurisdictional claims in published maps and institutional affiliations.

Ready to submit your research? Choose BMC and benefit from:

- fast, convenient online submission

- thorough peer review by experienced researchers in your field

- rapid publication on acceptance

- support for research data, including large and complex data types

- gold Open Access which fosters wider collaboration and increased citations

- maximum visibility for your research: over $100 \mathrm{M}$ website views per year

At $\mathrm{BMC}$, research is always in progress.

Learn more biomedcentral.com/submission 